\title{
Evolving Technologies and Standards Regulation
}

\author{
Luís Cabral* \\ New York University \\ David Salant* \\ Toulouse School of Economics
}

This draft: April 2008

\begin{abstract}
Spectrum regulation necessarily involves some regulation of the technology that licensees can use. One commonly stated assertion is that a mandated single standard, the solution followed by the EU for $2 \mathrm{G}$ wireless, is a successful model for spectrum regulation. We argue that a single standard leads to a free riding problem, and thus to a significant decrease in marginal incentives for R\&D investment. In this context, keeping two separate standards may be a necessary evil to sustain a high level of R\&D expenditures. We also provide conditions such that a non-standardization equilibrium is better for consumers and for society as a whole.
\end{abstract}

${ }^{*}$ Cabral: lcabral@stern.nyu.edu. Salant: dsalant@gmail.com. We are grateful to Jorge Padilla, Vasco Santos, and various seminar participants for useful comments and suggestions. The usual disclaimer applies. 


\section{Introduction}

Spectrum regulation necessarily involves some regulation of the technology that licensees can use. Beginning in the early 1990's, the US Federal Communications Commission (FCC), as well as regulatory agencies in other countries, have taken an increasingly laissez-faire approach to determining standards for wireless communications. For the Personal Communications Services (PCS) spectrum auctions, the FCC, as well as Industry Canada and the Mexican CFT, all allowed winning bidders to deploy any technology compatible with the band plan, power and emissions restrictions. At one point there were $2 \mathrm{G}$ technologies with virtually nationwide coverage in the US. In contrast, Europe, mandated that all firms allocated $2 \mathrm{G}$ spectrum licenses deploy only the GSM technology. For 3G, there were two main technologies deployed. However, despite significant pressure from the US government and from US firms, the EU mandated a single $3 \mathrm{G}$ standard. The EU seems to be taking a similar approach toward mobile television.

One commonly stated assertion is that the EU mandate of a single standard is a very successful model for spectrum regulation. However, economic analysis of this assertion is limited, and neither theory nor econometric evidence provide unambiguous support for it. The purpose of this paper is to formally examine the claim that standards regulation is welfare enhancing. We develop a model featuring non-cooperative $R \& D$ competition and cooperative standard setting. Contrary to the above view, we find that, under some circumstances, standards competition results in higher consumer surplus and social welfare than mandated standards. Moreover, market based standards generally result in faster innovation than standards regulation.

More specifically, we consider a world in which the relative quality of each standard evolves over time as a result of each firm's R\&D expenditure. We argue that standardization - at least early standardization - leads to a free riding problem, and thus to a significant decrease in marginal incentives for R\&D investment. In this context, keeping two separate standards may be a necessary evil to sustain a high level of R\&D expenditures. Specifically, we consider a model such that myopic firms would always agree to standardization; but considering the dynamics of product innovation, in equilibrium firms opt for developing their own standard. We also provide conditions such that a non-standardization equilibrium is socially optimal.

- Related literature. Several authors have dealt with the the economic 
analysis of standard setting. Perhaps closest to our paper, Choi (1996) considers the trade-off between the short-run benefits from standardization and the long-run benefits from experimenting with different technologies. Under certain conditions, he finds that ex post standardization is optimal (as is the case in our paper).

Nisvan and Minehart (2007) present a multi-period model of $R \& D$ with the possibility of firms sharing technology. Their setup is different from ours (for example, no profits are earned until all $n$ steps of R\&D are successfully completed; and the possibility of firm exit is explicitly considered). Moreover, their focus is also somewhat different (less on the benefits from standardization, more on the costs of product market collusion).

The paper is organized as follows. In Section 2, we outline some of the main milestones in the history of wireless telephony, with an emphasis on the process of standard setting and the persistent lack of a single standard. In Section 3, we introduce a model of $\mathrm{R} \& \mathrm{D}$ and standards setting and the main result of our paper: there are situations when, despite costless bargaining and market benefits from standardization, the equilibrium features multiple, incompatible standards. Section 4 extends the analysis to consider social welfare. We provide conditions such that an equilibrium with multiple standards is socially optimal. Section 5 provides a discussion of the main results and Section 6 concludes the paper.

\section{History of wireless standards competition}

Wireless telecommunications have a long history of standardization issues. First generation $(1 \mathrm{G})$ wireless mobile voice (and data) communications came under two different standards: Analog Mobile Phone System (AMPS) and the Nordic Mobile System (NMTS). AMPS was the mandatory North American standard. Most of the rest of the world, including Europe, was split between AMPS and NMTS (Gandal, Salant, and Waverman, 2003).

Starting in the early 1980s, four different second generation $(2 \mathrm{G})$ standards were introduced: GSM (often called Global System for Mobile Communications); TDMA (time division multiple access); iDEN; and CDMA (code division multiple access). GSM, TDMA and iDEN all divide a carrier channel into time slots, and digitally encode the signal on the time slots; they differ in the time division protocols used. CDMA, the latest standard to be developed, can usually pack more bits, or voice calls, into a given 
amount of spectrum than can GSM or TDMA. ${ }^{1}$

The European Union delegated standard setting to the European Technical Standards Institute (ETSI), which mandated GSM. In contrast, the FCC in the U.S. and regulators in other countries, including Australia, China India, and various South American countries have allowed operators to select their own standards based on economic or whatever criteria they wanted (Gandal, Salant, and Waverman, 2003; Cabral and Kretschmer, 2006). As a result, virtually all 2G networks in Europe are GSM, while elsewhere either the European policy was followed or there are competing standards. In the U.S., for example, GSM was the first $2 \mathrm{G}$ standard deployed (by Sprint in Washington, DC). TDMA and CDMA were introduced shortly thereafter (the latter by Sprint, GTE, Primeco, Bell Atlantic-NYNEX (all but Sprint are now part of Verizon Wireless) and Ameritech, among others).

At an early point of the development of third generation wireless (3G), there was a tentative accord for a single $3 \mathrm{G}$ standard. However, a number of European equipment vendors who dominated ETSI (namely Ericsson, Nokia and Siemens) decided on a variation of the original 3G standard, CDMA2000, which was developed by QUALCOMM. As ETSI sets standards policy for spectrum in the EU, European operators adopted a slight variation of the CDMA2000 standard, namely WCDMA. ${ }^{2}$ We thus have two 3G standards: CDMA2000 (or CDMA1X), created by QUALCOMM; and WCDMA, the ETSI derived standard.

CDMA2000 is essentially an upgrade of second generation CDMA and is largely backwards compatible. WCDMA (also called UMTS) is a variation of the CDMA2000 standard. It is essentially incompatible with either CDMA2000 or second generation CDMA (Salant and Waverman, 1998, 1999). What we mean by incompatible is that handsets meant to work on one standard will not easily work on the other one. In addition, 2G CDMA operators can easily upgrade to CDMA2000, merely by replacing some radio equipment at base stations and upgrading the software in the switches. By contrast, 2G CDMA operators cannot easily upgrade to WCDMA. Finally, for GSM operators the cost of upgrading to CDMA2000 or WCDMA

1. iDEN was deployed specifically for refarming narrow slivers of spectrum previously used for trunk radio service.

2. The U.S. was able to successfully lobby the EU to reverse the decision to require all European Union national regulatory agencies (NRAs) to require the ETSI mandated standard (as this would be a violation of a US/EU competition policy treaty). However, the EU NRAs allocated frequency in a way that makes it difficult for anyone to deploy CDMA2000; and in fact no firm has done so in the 3G bands. 
is about the same.

From a non-traveling-user point of view, the costs of multiple incompatible standards may not be very significant. In fact, every user has universal access to other users, regardless of which network they are connected to. There may be connection charges, but these result from there being more than one network, not from there being more than one standard. A traveling user may incur additional costs insofar as roaming may be limited. For example, a U.S. user with a CDMA or CDMA2000 handset will not be able to use it in Europe. However, many GSM handsets that are sold to European users can also be used in the U.S.

The costs of multiple standards would then seem to be primarily borne out by operators and equipment manufacturers. For example, the market for GSM handsets and terminal equipment is greater than that for CDMA based equipment, allowing for greater economies of scale in the former. For a chipset manufacturer like QUALCOMM, lack of standardization in 3G implies additional costs for various reasons: in addition to the loss of scale economies, a portion of its CDMA software must be re-written to work in WCDMA.

The above history of the wireless telecommunications industry leads to the puzzling question which motivates our analysis: If multiple standards create additional costs (for equipment manufacturers, operators and users), then why don't we observe an agreement on a single standard? Why the secession by Ericsson, Nokia and Siemens, which seems counter to the lock-in predicted by typical models of standards setting? One possible answer relies on the inefficiencies of negotiations among multiple players with possibly conflicting goals. In this paper, we argue that lack of standardization may be the natural outcome of competition even in a world with no inefficiencies in negotiations (Section 3); and may in fact be the socially optimal outcome (Section 4).

\section{$3 \quad$ Model and equilibrium results}

Consider an infinite horizon duopoly in an industry with an evolving technology. Specifically, suppose a technology can be at two different levels: $0,1 .^{3}$ The horizon is divided into discrete periods, each of which is divided

3. In terms of our wireless story, we can interpret level 0 as $2 \mathrm{G}$ and level 1 as $3 \mathrm{G}$. Later, we may want to consider the extension to three levels, which is not trivial and adds some new elements. 


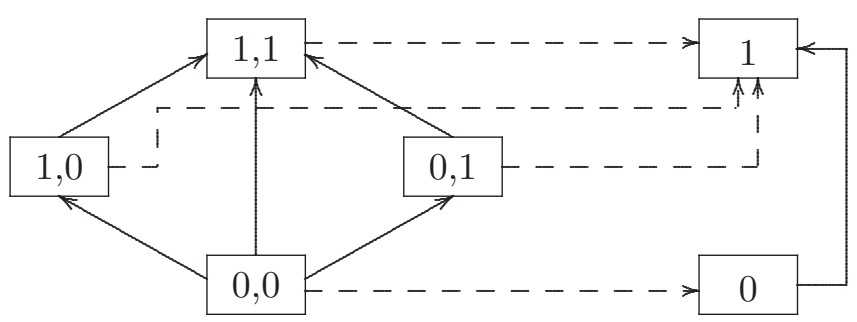

Figure 1: State space and transition paths. Solid arrows represent transitions by means of innovation outcomes. Dashed arrows represent transitions by means of standardization agreements.

into three stages. In a first stage, firms decide whether to make their technology designs compatible. In a second stage, firms independently make an R\&D investment towards improving their technology. Specifically, in order to innovate with probability $\rho$ a firm must spend $\frac{1}{2} \rho^{2} .4$ Finally, in a third stage product market profits for the period are received.

In each period, the state of the game can be described by two elements: the status of standardization and the technology level. Suppose that standardization has not yet been achieved. Then the sate of the game is summarized by the technology level of each firm. We then denote the state by ${ }_{D}(i, j)$, where ${ }_{D}$ stands for dual standards. If standardization has been achieved, then the state of the game is given by ${ }_{S}(i)$, where ${ }_{S}$ stands for single standard and $i$ is the (common) technology level. ${ }^{5}$

Figure 1 summarizes the state space. Each rectangle represents a state. States with two numbers (left-hand side of the figure) represent dual standard states; states with one number (right-hand side of the figure) represent single standard states. Solid arrows represent transitions by means of innovation outcomes. Dashed arrows represent transitions by means of standardization agreements. Our assumptions regarding transition between states are formalized as follows:

Assumption 1 (i) Starting from state ${ }_{D}(i, j)$, an improvement in firm i's technology leads to state ${ }_{D}(i+1, j)$. (ii) Starting from state ${ }_{S}(i)$, an im-

4. Naturally, if a firm is at technology level 1 it will not spend any resources on innovation.

5. To be rigorous, the state of the game must also include an indication of what part of the period is being played, in particular, whether standard negotiations have taken place. However, it should be clear from the context what particular moment we are considering. 
provement in any firm's technology leads to state ${ }_{S}(i+1)$. (iii) Starting from state ${ }_{D}(i, j)$, a standardization agreement leads to state ${ }_{S}(\max \{i, j\})$.

We thus consider two forms of state transitions. The first one is $R \& D$, and it works according to Assumption 1 (i) and (ii). The second form of state transition corresponds to standardization agreements, and it works according to Assumption 1 (iii). ${ }^{6}$ Notice that technology transitions take one period, whereas standardization agreements are instantaneous.

Having defined the state of the game, we now let $V_{D}(i, j)$ and $V_{S}(i)$ be firm $i$ 's value function (in a state with dual and single standard, respectively). That is, these are the value functions for the firm whose technology is at level $i$. Let $\pi_{D}(i, j), \pi_{S}(i)$ be the corresponding per-period product market profit functions.

Our next assumption relates to the nature of the standardization process. Whereas R\&D effort choices are independently and non-cooperatively chosen, we assume the standardization process consists of a negotiation between the firms. Specifically, we assume efficient, equal-split bargaining: ${ }^{7}$

Assumption 2 If standardization is efficient, that is, if $2 V_{S}(\max \{i, j\})>$ $V_{D}(i, j)+V_{D}(j, i)$, then standardization takes place and the gains are equally split between the firms.

Our final assumption relates to the profit functions:

$$
\begin{array}{ll}
\text { Assumption } 3 & \text { (a) } \pi_{S}(i)>\pi_{D}(i, i), i=0,1 ; \\
\pi_{D}(0,1) \text {. }
\end{array}
$$

This implies that, at every possible state, product market industry profits are greater with standardization than without.

Before proceeding, it is worthwhile restating our basic assumptions. A critical part of Assumption 1 is that standardization is an "absorbing" state. That is, once firm agree on a standard, then whatever improvements are achieved to that standards are shared by both firms, that, both firms continue to own the common standard. This assumption play a crucial role in our results. Assumptions 2 and 3 are made primarily for expositional purposes. In fact, they stack the cards in favor of standardization (bargaining

6. Notice that we only consider two levels of technology development. Therefore, Definition 1 really only applies to $i=0$. Alternatively, we make the convention that state ${ }_{D}(i, j)$ is equivalent to stage ${ }_{D}(1, j)$ when $i>1$ (and the same for ${ }_{S}(i)$.)

7. To justify this, suppose that firms make alternating offers and that the time interval between offers in negligible. Rubinstein's (1982) result then implies our assumption. 
is efficient, standardization increases product market profits). By making these assumptions, it is easier to understand the nature of our result, namely that standardization may not take place in equilibrium.

Our first result in this section refers to the equilibrium level of R\&D effort:

Proposition 1 The equilibrium levels of R\&D are lower when standardization takes place. Specifically, $\rho_{i}$ and $\rho_{j}$ are higher in state ${ }_{D}(i, j)$ than in state $_{S}(\max \{i, j\})$.

The proof of this and other results in the paper may be found in the Appendix. Here we present the main intuition. An important step in the proof is to show that

$$
V_{D}(1,0)>V_{S}(1)>V_{D}(0,1) .
$$

Even though in equilibrium state ${ }_{D}(0,1)$ leads to state ${ }_{S}(1)$, that is, firms agree on a common standard, the ex-ante payoff is greater for the firm that owns the superior technology. This in turn implies that innovation incentives when both firms are at technology level 0 are different whether there is one standard or two standards. Consider the case when firm $i$ 's rival does not innovate. Then the the marginal gain from innovation by firm $i$ is greater under dual standards by $V_{D}(1,0)-V_{S}(1)$. If firm $i$ 's rival does innovate, then the extra marginal gain is given by $V_{S}(1)-V_{D}(0,1)$. Either way, the marginal gain is greater under no standardization.

Intuitively, under standardization any marginal improvement created by firm $i$ is available both to $i$ and its rival. This creates a free-riding problem (a positive externality on firm $j$ ). More importantly, it implies a private bad for firm $i$ : it suffers directly from the fact that firm $j$ improves along with firm $i$. Assumption 1(ii) thus plays a crucial role in Proposition 1. Even though firms continue to choose innovation effort independently, under standardization the benefits from innovation are equally shared by both firms.

The externality created by standardization suggests that firms may prefer not to standardize as a way to internalize innovation incentives. Our next result provides sufficient conditions for this to happen.

Proposition 2 Suppose that $\pi_{S}(0)-\pi_{D}(0,0)$ and $\pi_{D}(1,0)-\pi_{D}(0,1)$ are small. Then standardization occurs in all states ${ }_{D}(i, j)$ except ${ }_{D}(0,0)$. 


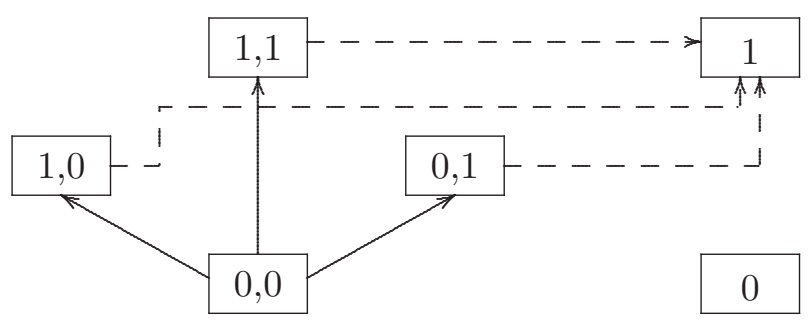

Figure 2: State space and transition paths observed along the equilibrium path (with positive probability).

It may seem surprising at first how general Proposition 1 is, whereas Proposition 2 requires several parameter assumptions (which however are sufficient, not necessary conditions). As mentioned above, standardization implies an externality: a benefit conferred on the rival firm. However, competitive R\&D implies itself an externality: part of the gain obtained by firm $i$ is gotten at the expense of firm $j$. Therefore, the fact that lack of standardization leads to higher levels of innovative effort (Proposition 1) does not necessarily imply that it is preferred by firms. Proposition 2 sets out a set of sufficient conditions such that the effect in Proposition 1 leads firms to agree not to standardize. The assumption that $\pi_{S}(1)-\pi_{S}(0)$ is large may be interpreted as meaning that innovation is valuable. The assumption that $\pi_{S}(0)-\pi_{D}(0,0)$ is small ensures that the short-term loss from lack of standardization is not too large. Finally, the assumption that $\delta$ is small implies that equilibrium $\rho$ is small and thus the business stealing effect is small.

Figure 2 summarizes Proposition 2. Specifically, it shows all transitions that are observed along the equilibrium path with positive probability. Since the game starts from state ${ }_{D}(0,0)$, we see that state ${ }_{S}(0)$ is never visited. As soon as one of the firms achieves level 1, standardization ensues.

\section{Social welfare}

Propositions 1 and 2 are about positive analysis. They provide conditions under which standardization does not take place in state $(0,0)$ even though firms' profits would be greater in every state if firms were to standardize. From the firms' point of view, the short-term losses from lack of standardization are more than compensated by longer-term benefits of higher levels of R\&D expenditure. In fact, from the firms' point of view the equilibrium 
pattern of standardization is optimal.

In order to go from industry profits to social welfare we need a more detailed model of product market competition and consumer welfare. Insofar as consumer welfare and industry profits are relatively aligned regarding standardization decisions, Proposition 2 can be extended to state that no standardization at stage $(0,0)$ is socially optimal. Whether this is true depends on the particular model we have in mind. In what follows, we present a particular model that we believe reasonably reflects some of the features of wireless communications.

Looking at the current situation of wireless communications in the U.S., we note that lack of standardization regarding the basic technology does not prevent consumers from benefiting from network effects: every consumer can communicate with every other consumer, regardless of which technology they are hooked up to. Lack of standardization can imply higher costs for sellers, who have to create means of hooking up networks based on different technologies. To the extent that these higher costs are reflected on prices, consumers are worse off. In other words, it seems fair to say that, when it comes to standardization, the main concern for consumers is prices rather than network effects. ${ }^{8}$

To be more specific, consider a Hotelling type duopoly where each firm is located at the extreme of a product variety segment and consumers are uniformly distributed along that segment (each consumer buys one unit from one of the sellers). If the sellers' technologies are not standardized, then both their fixed costs and their marginal costs must increase in order to provide consumers with universal network access. Let $k_{0}, c_{0}$ and $k_{1}, c_{1}$ be the sellers' fixed and marginal cost with and without standardization. Assume that $k_{0}>k_{1}$ and $c_{0}>c_{1}$. This is consistent with Assumption 3, namely that industry profits are greater under standardization. Specifically, equilibrium firm profits are given by

$$
\pi=\frac{1}{2} t-k_{i}(i=1,2)
$$

\footnotetext{
8. A single standard, can, but need not, provide consumers with better coverage, especially during the roll-out phase. The anecdotal evidence contrasting European and North American experience, without controlling for differences in dates of spectrum allocations and population density, suggests that coverage was better in Europe. However, firms offering competing standards can have stronger incentives to compete in coverage than those offering the same technology.
} 
whereas consumer surplus is given by

$$
\mu=v-t-c_{i}(i=1,2)
$$

where $v$ is consumer valuation and $t$ is the "transportation" cost.

Our main result in this section is that, if sellers' and buyers' incentives are properly aligned as regards the standardization decision, then the nostandardization equilibrium result from Proposition 2 can be extended to social welfare.

Proposition 3 If $c_{1}-c_{0}$ is sufficiently small, then if in equilibrium there is no standardization in state $(0,0)$ it follows that no standardization is the socially optimal outcome in that state.

\section{Discussion}

Our main result, Proposition 2, states that if the gains from innovation are significant and the short run benefits from standardization relatively small, then in equilibrium firms prefer to follow different paths in their R\&D efforts. What does this have to say regarding wireless telecommunications, the main motivating example we consider in this paper? We can think of third generation as our level zero technology and R\&D as the effort to reach $4 \mathrm{G}$. From a short run point of view, it might seem more efficient for Qualcomm and Nokia to agree on a common standard of $3 \mathrm{G}$, that is, to move to state $S_{S}(0)$. As it happened, the state remained at ${ }_{D}(0,0)$, with WCDMA and CDMA2000 representing each of the 0's. Many may lament this as an inefficient equilibrium resulting from inefficiencies in negotiations. We argue that, given the incompleteness of contracts involving IP, dual standards may have the benefit of maintaining research incentives that might otherwise be inefficiently diminished.

We take a somewhat extreme approach by assuming that, under standardization, all technology improvements are shared by the adherents to that standard; whereas, under dual standards, imitation is only possible under a standardization agreement. Reality is probably between these extremes. But to the extent that standardization increases the free-riding problem of R\&D effort, our qualitative result still holds.

We consider a simple framework with two technology levels, 0 and 1 . But before $3 \mathrm{G}$ there as $2 \mathrm{G}$; and after $4 \mathrm{G}$ there will likely be $5 \mathrm{G}$. We could consider a more general framework with an infinite technology ladder. Suppose 
that, in addition to standardization, firms may write license agreements. Our conjecture is that, each time a firm gets one step ahead of its rival, the laggard will license the technology from the leader but not follow the same standard. We then reach state ${ }_{D}(1,1)$, which effectively becomes the new state ${ }_{D}(0,0)$. Technology licensing then has the benefits of (efficiently) bringing all firms to a higher technology level without imposing the freeriding inefficiencies of standardization.

Our assumptions regarding short-run profits purposely stack the cards in favor of standardization (that is, in each period, profits under a single standard are greater than under dual standards). The same is not necessarily true regarding our assumption of efficient negotiations. If negotiation costs are prohibitively high, then trivially there is no standardization agreement. However, intermediate levels of negotiations costs may or may not favor standardization. If the cost is uniform across all possible instances, then negotiations costs favor no standardization to the extent that they delay the cost from standard setting negotiations. Specifically, $V_{S}(0)$ is decreased by $N$, the negotiation cost, whereas $V_{D}(0,0)$ is decreased by $\delta N$. If $N$ is large and $\delta$ small, this may switch the equilibrium from standardization to delayed standardization. However, it might be argued that the costs of reaching an agreement are higher when firms' technology levels are different than when they're equal. In that case, negotiations costs might favor early standardization. Specifically, $V_{S}(0)$ is decreased by $N_{S}$, whereas $V_{D}(0,0)$ is decreased by $\left(1-\rho^{2}\right) N_{A}+\rho^{2} N_{S}$, where $N_{S}$ is the negotiation cost when both firms are at the same technology level and $N_{A}$ is the negotiation cost when firms are at different technology levels. If the difference between $N_{S}$ and $N_{A}$ is sufficiently large, then the equilibrium may switch from delayed standardization to standardization in state $(0,0)$.

\section{Conclusion}

Our analysis indicates that a regulatory policy mandating a single standard can, at times, be harmful, both from a consumer and from social point of view. We provide a set of conditions such that, absent regulation, firms choose incompatible technologies. In this context, regulatory policy mandating compatible standards reduces investment incentives, retards innovation, and may ultimately reduce consumer and social welfare.

Our model suggests that the relation between standardization and innovation incentives is relatively robust. By contrast, the relation between a 
mandated standard and consumer welfare depends on various crucial parameters. If the consumer loss from multiple standards is sufficiently large, and if firm profits are not well aligned with consumer welfare, then a mandated standard may increase consumer welfare.

Finally, while our paper was motivated by the wireless telecommunications industry, we believe the problems we highlight are of more general importance. For example, the EU recently decided on a standard for mobile TV. On March 17, 2008, Viviane Reding, EU Commissioner for the Information Society and Media, stated that

For Mobile TV to take off in Europe, there must first be certainty about the technology. This is why I am glad that with today's decision, taken by the Commission in close coordination with the Member States and the European Parliament, the EU endorse DVB-H as the preferred technology for terrestrial mobile broadcasting. ${ }^{9}$

While we cannot claim the EU's decision to be right or wrong in the present context, we challenge the assertion that a mandated standard is in general the best solution.

9. See http://europa.eu/rapid/pressReleasesAction.do?reference=IP/08/451\&format= HTML\&aged=0\&language=EN\&guiLanguage=en, visited on April 7, 2008. 


\section{Appendix}

Proof of Proposition 1: Suppose we are in state ${ }_{D}(1,1)$, that is, both firms are at technology level 1 and each has its own standard. If firms do not agree on a common standard, then product market profits are $\pi_{D}(1,1)$ for each. If they agree on a common standard, then product market profits are $\pi_{S}(1)$ for each. Assumption 3 then implies that at state $(1,1)$ firms agree on a common standard. In fact, there is no additional $R \& D$ and so product market profits is all that matters; and, by assumption, we have efficient bargaining, which leads to the efficient solution (from the firms' perspective). We thus have

$$
V_{D}(1,1)=V_{S}(1)=\frac{\pi_{S}(1)}{1-\delta}
$$

where $\delta$ is the discount factor. Now suppose we are in state $D(1,0)$. Since firms can achieve the same industry payoffs as in state ${ }_{S}(1)$, and given Assumptions 2 and 3, we again conclude that firms choose standardization. We thus have $V_{D}(0,1)+V_{D}(1,0)=2 V_{S}(1)$. The exact split of the pie $2 V_{S}(1)$ depends on the outside option for each firm, which we consider next.

Let $\widetilde{V}_{D}(0,1)$ and $\widetilde{V}_{D}(1,0)$ denote the value function corresponding to a one-time deviation from the equilibrium path whereby firms do not agree on a common standard. That is, $\widetilde{V}_{D}(0,1)$ corresponds to the outside option in the standardization negotiations. We then have

$$
\begin{aligned}
& \widetilde{V}_{D}(0,1)=\pi_{D}(0,1)+\delta\left(\rho \frac{\pi_{S}(1)}{1-\delta}+(1-\rho) V_{D}(0,1)\right)-\frac{1}{2} \rho^{2} . \\
& \widetilde{V}_{D}(1,0)=\pi_{D}(1,0)+\delta\left(\rho \frac{\pi_{S}(1)}{1-\delta}+(1-\rho) V_{D}(1,0)\right) .
\end{aligned}
$$

(Recall that the leading firm chooses zero research effort.)

We are now ready to analyze the negotiation game at stage ${ }_{D}(0,1)$. If there is standardization, then each firm gets $\pi_{S}(1)$. If negotiations break down, then firms get $\widetilde{V}_{D}(0,1)$ and $\widetilde{V}_{D}(1,0)$. We then have

$$
\begin{aligned}
& V_{D}(0,1)+V_{D}(1,0)=2 V_{S}(1) \\
& V_{D}(0,1)-\widetilde{V}_{D}(0,1)=V_{D}(1,0)-\widetilde{V}_{D}(1,0)
\end{aligned}
$$

The first equation follows from Assumption 2 (efficient bargaining) and Assumption 3 (standardization increases joint profits). The second equation 
states that the gains from standardization are equally split between the two firms (again by Assumption 2). Solving the above system of equations, we get

$$
\begin{aligned}
& V_{D}(0,1)=V_{S}(1)-\Delta \\
& V_{D}(1,0)=V_{S}(1)+\Delta .
\end{aligned}
$$

where

$$
\Delta \equiv \widetilde{V}_{D}(1,0)-\widetilde{V}_{D}(0,1)
$$

Subtracting (2) from (3) and simplifying, we get

$$
\Delta=(\delta(1-\rho))^{-1}\left(\pi_{D}(1,0)-\pi_{D}(0,1)+\frac{1}{2} \rho^{2}\right)>0 .
$$

Together with (4), this implies that

$$
V_{D}(0,1)>V_{S}(1)>V_{D}(0,1) .
$$

Let us now consider state ${ }_{D}(0,0)$ and and suppose firms decide to standardize. Then each firm maximizes

$$
V_{S}(0)=\pi_{S}(0)+\delta\left((1-\rho)(1-\widetilde{\rho}) V_{S}(0)+(1-(1-\rho)(1-\widetilde{\rho})) V_{S}(1)\right)-\frac{1}{2} \rho^{2},
$$

where $\widetilde{\rho}$ is the rival's R\&D effort. (Recall that we make the assumption that, if firms agree on a standard, then all future technology improvements are shared.) Suppose instead that firms decide not to standardize. Then each firm maximizes

$$
\begin{gathered}
V_{D}(0,0)=\pi_{D}(0,0)+\delta\left((1-\rho)(1-\widetilde{\rho}) V_{D}(0,0)+\rho(1-\widetilde{\rho}) V_{D}(1,0)+\right. \\
\left.+(1-\rho) \widetilde{\rho} V_{D}(0,1)+\rho \widetilde{\rho} V_{D}(1,1)\right)-\frac{1}{2} \rho^{2}
\end{gathered}
$$

Define

$$
\Phi \equiv(\delta(1-\delta(1-\rho)(1-\widetilde{\rho})))^{-1}\left(\frac{\partial V_{D}(0,0)}{\partial \rho}-\frac{\partial V_{S}(0)}{\partial \rho}\right) .
$$

Taking into account that $V_{D}(1,1)=V_{S}(1)$, computation establishes that

$$
\Phi=(1-\widetilde{\rho})\left(V_{D}(1,0)-V_{D}(1,1)\right)+\widetilde{\rho}\left(V_{D}(1,1)-V_{D}(0,1)\right)>0,
$$


since, by $(6), V_{D}(1,0)-V_{D}(1,1)>0$ and $V_{D}(1,1)-V_{D}(0,1)>0$. It follows that the value of $\rho$ is greater under no standardization than under standardization.

Proof of Proposition 2: Consider a hypothetical equilibrium where firms agree to a common standard at $(0,0)$. From $(7)$, we see that, at a symmetric equilibrium, the derivative of firm value with respect to effort is given by

$$
\frac{\partial V_{S}(0)}{\partial \rho}=(1-\rho)\left(V_{S}(1)-V_{S}(0)\right)-\rho
$$

The increase in firm value following a joint increase in effort is in turn given by

$$
\frac{\partial V_{S}(0)}{\partial \rho}=2(1-\rho)\left(V_{S}(1)-V_{S}(0)\right)-\rho
$$

which is greater. In words, we have the classic free-rider problem whereby equilibrium effort is (strictly) less than jointly efficient level.

Consider now a one-time deviation whereby firms do not agree on a standard at $(0,0)$ in the current period. (That is, if none of the firms succeeds in the current period, then next period firms agree on a common standard even though we are still in state $(0,0)$.)

If firms fail to agree on a standard, then each firm will be maximizing

$$
\begin{aligned}
V_{D}^{\circ}(0,0)=\pi_{D}(0,0)+\delta((1-\rho) & (1-\widetilde{\rho}) V_{S}(0)+\rho(1-\widetilde{\rho}) V_{D}(1,0)+ \\
& \left.+(1-\rho) \widetilde{\rho} V_{D}(0,1)+\rho \widetilde{\rho} V_{D}(1,1)\right)-\frac{1}{2} \rho^{2} .
\end{aligned}
$$

If $\pi_{S}(0)$ is close to $\pi_{D}(0,0)$, then the difference in discounted payoff between standardization and no standardization at $(0,0)$ results from differences in the value of $\rho$. If moreover, $\pi_{D}(0,1)$ is close to $\pi_{D}(1,0)$, then $V_{D}(0,1) \approx V_{D}(1,0) \approx V_{S}(1)$, and the equilibrium value of $\rho$ is approximately the same as the equilibrium value resulting from (7). In words, no standardization implies a small increase in the value of $\rho$ (we know it's an increase by Proposition 1).

Finally, by the argument at the beginning of the proof, an increase in research effort increases the value of firms. Since bargaining is efficient, firms will agree on not having a common standard, thus increasing each firm's payoff. 
Proof of Proposition 3: If $c_{1}-c_{0}$ is sufficiently close to zero, then most of the cost of providing connection under no standardization is borne out by sellers. Since we assume efficient bargaining between sellers, the equilibrium outcome is optimal from the sellers' point of view, which in turn is a sufficient condition for it to be better from a social point of view. 


\section{References}

Cabral, Luís M B, and Tobias Kretschmer (2006), "Standards Battles and Public Policy," in S Greenstein and V Stango (Eds), Standards and Public Policy, Cambridge: Cambridge University Press.

Chor, JaI-Pil (1996), "Standardization and Experimentation: Ex Ante vs. Ex Post Standardization," European Journal of Political Economy 12, 273-290.

Erkal, Nisvan, and Deborah Minehart (2007), "Optimal Sharing Strategies in Dynamic Games of Research and Development," Department of Justice Working Paper EAG 07-7, April 2007.

Gandal, NeIl (2002), "Compatibility, Standards and Network Effects: Some Policy Implications," Oxford Review of Economic Policy 18:1, 80-91.

Gandal, Neil, David Salant, and Leonard Waverman (2003), "Standards in Wireless Telephone Networks," Telecommunications Policy 27, 325-332.

Salant, David, and Leonard Waverman (1998), "The Use of Standard Setting as a Mean of Facilitating Cartels and its Trade Effects," International Competition Policy Advisory Committee, U.S. Department of Justice, November.

Salant, David, And Leonard Waverman (1999), Testimony at International Competition Policy Advisory Committee, U.S. Department of Justice, May.

Saloner, Garth (1998), "Economic Issues in Computer Interface Standardization," Economics of Innovation of New Technlogy 1, 135-156.

Yavuz, Emre A, and Victor Leung (2002), "A Comparison Study of 3G System Proposals: CDMA2000 vs. WCDMA," Proceedings of the IASTED International Conference on Wireless and Optical Communications, Banff, AB, pp. 62-67. 